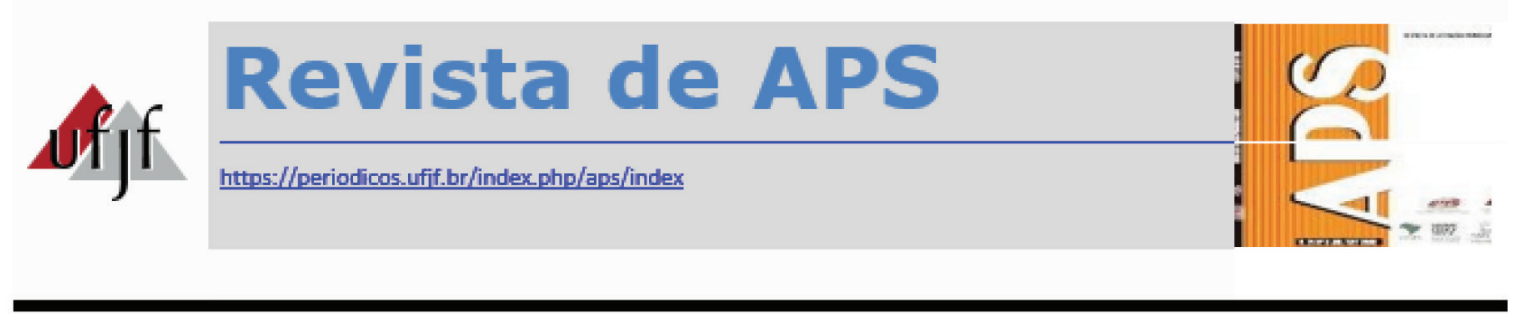

\title{
Mapeamento da rede de atenção ao idoso de uma região de Juiz de Fora, Minas Gerais
}

\author{
Mapping of the elderly care network of a region from Juiz de Fora, Minas \\ Gerais
}

\author{
Elaine Regina Pereira Carvalho ${ }^{1}$, Jordania Lindolfo Almas², Maria Alice Junqueira \\ Caldas $^{3}$
}

\begin{abstract}
RESUMO
Introdução: O envelhecimento populacional é considerado um dos maiores desafios da Saúde Pública, principalmente nos países em desenvolvimento. É relevante conhecer a rede de atenção para que possam ser elaboradas políticas, programas e ações que atendam as suas especificidades. $\mathrm{O}$ objetivo foi identificar e mapear a rede de atenção à população idosa da região Norte de Juiz de Fora (MG), com a finalidade de discutir estratégias de ação. Metodologia: Tratou-se de um estudo descritivo que identifica os serviços ofertados aos idosos através de três etapas: listagem da rede de saúde; entrevistas com um representante; e uso de tecnologias de acesso livre da internet para mapear e catalogar os serviços ofertados. Resultados: De acordo com o Catálogo Social de Juiz de Fora, 30 serviços disponibilizam atividades de forma direta ou indiretamente; na internet foram encontradas cinco entidades que ofertam algum serviço, totalizando 35 locais que foram foco da pesquisa. Destes, 24 são serviços governamentais e 11 não governamentais, e 16 são da rede de saúde. Discussão: Dos serviços encontrados, a maioria era composta por serviços de saúde, coordenada pela atenção primária, indicando a necessidade de um sistema que tenha esta como coordenadora de outros serviços de saúde. Foi observado que as atividades estão frequentemente voltadas para ações individuais, com pouco enfoque para a coletividade, ações de prevenção de agravos e promoção de saúde. Considerações Finais: Espera-se que este estudo

\footnotetext{
${ }^{1}$ Possui graduação em Fisioterapia pela Universidade Federal de Juiz de Fora (MG), pós-graduação lato sensu em Fisioterapia Neurológica Adulta pela Faculdade Unyleya / WPós e em Avanços Clínicos em Fisioterapia na área de Geriatria e Gerontologia pela Universidade Federal de Minas Gerias. Universidade Federal de Juiz de Fora. E-mail: elainerpk@hotmail.com

2 Fisioterapeuta com especialização em Fisioterapia Gerontológica Geriátrica. Universidade Federal de Juiz de Fora.

${ }^{3}$ Doutorado em Saúde Coletiva pela Universidade do Estado do Rio de Janeiro, Brasil(2006). Professor

Associado III da Universidade Federal de Juiz de Fora.
} 
possa contribuir para futuros trabalhos que mobilizem a sociedade organizada e o Estado, no sentido de dar respostas às necessidades da população idosa, que tende a crescer ainda mais.

PALAVRAS-CHAVE: Idoso. Assistência à saúde. Mapeamento geográfico.

\section{ABSTRACT}

Introduction: Population aging is considered one of the major challenges of Public Health, especially in developing countries. It is relevant to know the network of attention so that policies, programs and actions can be elaborated that meet their specificities. The objective was to identify and to map the network of attention to the elderly population of the northern region of Juiz de Fora (MG), in order to discuss strategies of action. Methodology: It was a descriptive study that identifies the services offered to the elderly through three stages: health network listing; Interviews with a representative; the use of free internet access technologies to map and to catalog the offered services. Results: According to the Juiz de Fora Social Catalog, 30 services provide activities directly or indirectly; On the Internet, five entities that offer some service were found, totaling 35 places that were the focus of the research. Of these, 24 are governmental services, 11 are non-governmental, and 16 are from the health network. Discussion: Among the services found the majority was composed of health services, coordinated by primary care indicating the need for a system that has it as coordinator of other health services. It was observed that activities are often focused on individual actions, with little focus on the community, and actions to prevent worsening and to promote health. Final Considerations: It is hoped that this study may contribute to future works that mobilize the organized society and the State, in order to respond to the needs of the elderly population, which tends to grow even more.

KEYWORDS: Aged. Delivery of Health Care. Geographic Mapping.

\section{INTRODUÇÃO}

O envelhecimento populacional é considerado um dos maiores desafios da Saúde Pública, principalmente nos países em desenvolvimento ${ }^{1}$. Esse processo acontece de forma rápida, sem tempo para uma reorganização social e da saúde adequada para atender às novas demandas emergentes ${ }^{2}$. Essa mudança pode ser provada pela alteração do formato das pirâmides populacionais, demonstrada pelo aumento do topo que se correlaciona à população idosa e o estreitamento da base que corresponde às pessoas mais jovens ${ }^{3}$. Apesar dos avanços tecnológicos que possibilitaram esse crescimento, não houve investimentos em políticas públicas, criando um "envelhecimento artificial" sem qualidade de vida4.

A Organização Mundial de Saúde (OMS) conceitua como idoso a pessoa acima de 60 anos, para países em desenvolvimento. No Brasil, essa definição foi confirmada pela lei que dispõe sobre a Política Nacional do Idoso ${ }^{3}$. Um país em desenvolvimento é classificado como envelhecido quando a população de 60 anos ou mais excede $7 \%$ do total $^{5}$. Em 2013, o grupo etário dos idosos correspondeu a 13,0\% da população total ${ }^{6}$. Em relação às proporções de idosos nas regiões do Brasil, ocorre um aumento 
mais significativo na região Sudeste, e menor na região Norte ${ }^{3}$. Especificamente em Juiz de Fora-MG, de acordo com o CENSO 2010, a população total é de 516.247, sendo a população idosa de 70.288 (13,62\%). Entre os anos de 2000 a 2010, a faixa etária que mais cresceu no município foi a de idosos, alcançando um índice de 45,6\%7.

A maioria da população idosa possui doenças e agravos crônicos não transmissíveis (DNT), que são estados permanentes ou de longa permanência, que requerem acompanhamento constante e frequentemente estão associados a outras condições incapacitantes, que geralmente tendem a comprometer de forma significativa a qualidade de vida ${ }^{8,9,10}$. Conforme dizem Ursine et al. ${ }^{11}$, a ocorrência de doenças crônicas em um indivíduo juntamente com uma abordagem ineficiente do serviço de saúde levam à incapacidade e à dependência do idoso, afetando sua funcionalidade. Em geral, resulta na restrição das atividades de vida diária (AVD) ao ambiente domiciliar. A dependência é criada pela incapacidade somada à necessidade de cuidado, e esta é resultante da dificuldade de realização de funções sem auxílio de equipamentos ou pessoas ${ }^{12}$. Figueiredo et al. ${ }^{13}$ afirmaram que "com o acelerado envelhecimento da população, certamente crescerá o número de idosos acamados, fragilizados e com elevado grau de dependência e com várias necessidades básicas afetadas".

Quando ocorrem doenças incapacitantes, observa-se a necessidade de um cuidado contínuo ${ }^{12}$. O sistema de suporte informal tem crescido nos países em desenvolvimento, e este ocorre quando não há suporte formal proporcionado por profissionais capacitados, financiados pela família ou pelo governo. Com isso, a presença de cuidador-familiar se torna cada vez mais frequente, principalmente quando se trata de um idoso acamado. Em sua maioria, o cuidado torna-se permanente, exercido por mulheres e idosos mais jovens ${ }^{14}$.

Segundo o Ministério da Saúde ${ }^{8}$, o contato preferencial dos usuários com os sistemas de saúde se faz por meio das Unidades de Atenção Primária à Saúde (UAPS), e estas devem orientar-se pelos princípios da universalidade, da acessibilidade e da coordenação do cuidado, do vínculo e continuidade, da integralidade, da responsabilização, da humanização, da equidade e da participação social. Na Atenção Primária à Saúde (APS), é essencial desenvolver um diagnóstico dos grupos populacionais de cada área de abrangência para o planejamento de ações específicas de intervenções em saúde ${ }^{15}$.

Como forma de atenção domiciliar da rede de saúde, há a visita ao domicílio dos idosos que não têm acesso direto a UAPS, tornando importante para ampliar a visão do profissional quanto às condições reais da família, proporcionando atenção integral à saúde com ações de educação e investigação diagnóstica ${ }^{16}$. Sendo assim, essa assistência possibilita cumprir todos os princípios do Sistema Único de Saúde (SUS) levando em conta a singularidade do indivíduo. Para ter uma atenção domiciliar, é necessário que o profissional, idoso, cuidador e familiar tenham uma interação gerando um benefício para todos envolvidos ${ }^{17}$. 
Segundo Chaimowicz et $a l .{ }^{18}$, os idosos estão desamparados pelo sistema público de saúde, refletindo nas doenças existentes que geram incapacidades e perda de qualidade de vida. Diversas condições de saúde passam despercebidas pelo sistema, ocorrendo a ausência ou a incoerência de diagnósticos. Além disso, ainda existem poucos estudos representativos sobre essa faixa etária ${ }^{19}$. É relevante conhecer a rede de atenção, para que possam ser elaboradas políticas, programas e ações que atendam as suas especificidades. A atenção à saúde quando está direcionada para as características de uma comunidade deve englobar as necessidades específicas da população e gerar o cuidado de acordo com o contexto cultural e suas preferências ${ }^{20}$.

A rede de atenção envolve organizações com objetivos comuns propondo uma atenção contínua e integrada para um público específico ${ }^{20}$. Segundo Alvarenga ${ }^{21}$, é essencial a rede de suporte própria para o idoso, classificada em formal, como Instituição de Longa Permanência para Idosos (ILPI), atendimento domiciliar e hospitais, ou informal, que inclui serviços de inserção comunitária e práticas sociais. Um suporte adequado envolve, além do idoso, a sua família e seu cuidador.

O objetivo deste estudo foi identificar e mapear a rede de atenção específica para o idoso ou o cuidador da região Norte de Juiz de Fora.

\section{MATERIAIS E MÉTODOS}

O município de Juiz de Fora se localiza na mesorregião geográfica da Zona da Mata Mineira, com aproximadamente 500 mil habitantes. Seu território está dividido em sete regiões urbanas administrativas ${ }^{22}$, sendo a região Norte a de maior extensão territorial e, por isso, selecionada para o estudo.

Tratou-se de um estudo de caráter descritivo, com delineamento transversal. Foi considerado para este estudo que rede de atenção ao idoso é todo serviço de assistência direta que possa contribuir para o cuidado do idoso, seja governamental ou não, incluindo a atenção ao cuidador de idosos, ofertando, por exemplo, doação de fraldas geriátricas por uma igreja, doação de cesta básica pelo Centro de Referência de Assistência Social (CRAS). Sendo assim, como amostra do mapeamento da rede de atenção, estarão incluídas instituições do campo da saúde e social.

\section{Descrição das etapas da coleta dos dados}

Identificaram-se os diversos serviços ofertados à população idosa na zona Norte da cidade de Juiz de Fora, através de três etapas: 1a) listagem da rede de apoio por meio do contato com a Gestão Municipal, a partir da autorização para realização da pesquisa pela Secretaria de Saúde de Juiz de Fora, e entidades sociais, pesquisadas através do Catálogo Social do Município ${ }^{23}$ e da internet em pesquisa no Google com 
as palavras-chave "serviço de atenção ao idoso" e "Juiz de Fora"; 2aa) entrevistas, para o que primeiramente foi feito um contato por telefone para posterior agendamento das visitas nas instituições governamentais e não governamentais dessa região, onde o responsável pela instituição foi solicitado a assinar o Termo de Compromisso Livre e Esclarecido e responder a um questionário sobre a existência de algum serviço; e 3ạ) uso de tecnologias de acesso livre da internet com a finalidade de mapear e catalogar os serviços ofertados a essa população.

Na segunda etapa, o questionário contempla a descrição das atividades, a forma de acesso, o custo do serviço e a população-alvo. Para a terceira etapa, os métodos foram baseados na dissertação de mestrado de Costa ${ }^{24}$, que utilizou "ferramentas online de geocodificação" acessadas por um computador, como Google Fusion Tables e o formulário (Form), que são ferramentas do Google Drive acessado através de uma conta no e-mail da Google (Gmail), o acesso ao Google Earth, que localiza e referencia as coordenadas geográficas e imagens do Google Maps. Após a identificação dos serviços, que foram preenchidos no formulário (Form), ocorreu a marcação destes com símbolos plotados em um mapa de localização criado no Google Fusion Tables. Também foi criado um mapa de calor (heatmap), sendo demonstrada, a partir de cores, a concentração e dispersão dos serviços. Posteriormente, foi produzido um catálogo dessas redes sociais.

\section{Aspectos éticos}

O estudo foi aprovado pelo Comitê de Ética em Pesquisa Humana da Universidade Federal de Juiz de Fora, de acordo com a Resolução do Conselho Nacional de Saúde número 466/12 e parecer número 1.615.209.

\section{RESULTADOS}

De acordo com o Catálogo Social do município ${ }^{23}$, foram identificados 88 serviços socioassistenciais e dois em fase de implementação. Destes, apenas 30 disponibilizavam serviços direta ou indiretamente à população idosa da região Norte, como: atendimento médico, visitas domiciliares, doação de alimentos ou roupas, atividades físicas supervisionadas, grupos de orientações, entre outros. No levantamento feito utilizando a internet, foram encontradas 12 entidades, sendo que apenas cinco destas ofertavam algum serviço, de acordo com a Figura 1. Nestes 35 locais, foram feitas as entrevistas e aplicado o questionário. A pesquisa foi finalizada em agosto de 2016. 
Figura 1 - Processo de levantamento da rede de assistência à população idosa da região Norte de Juiz de Fora, Minas Gerais

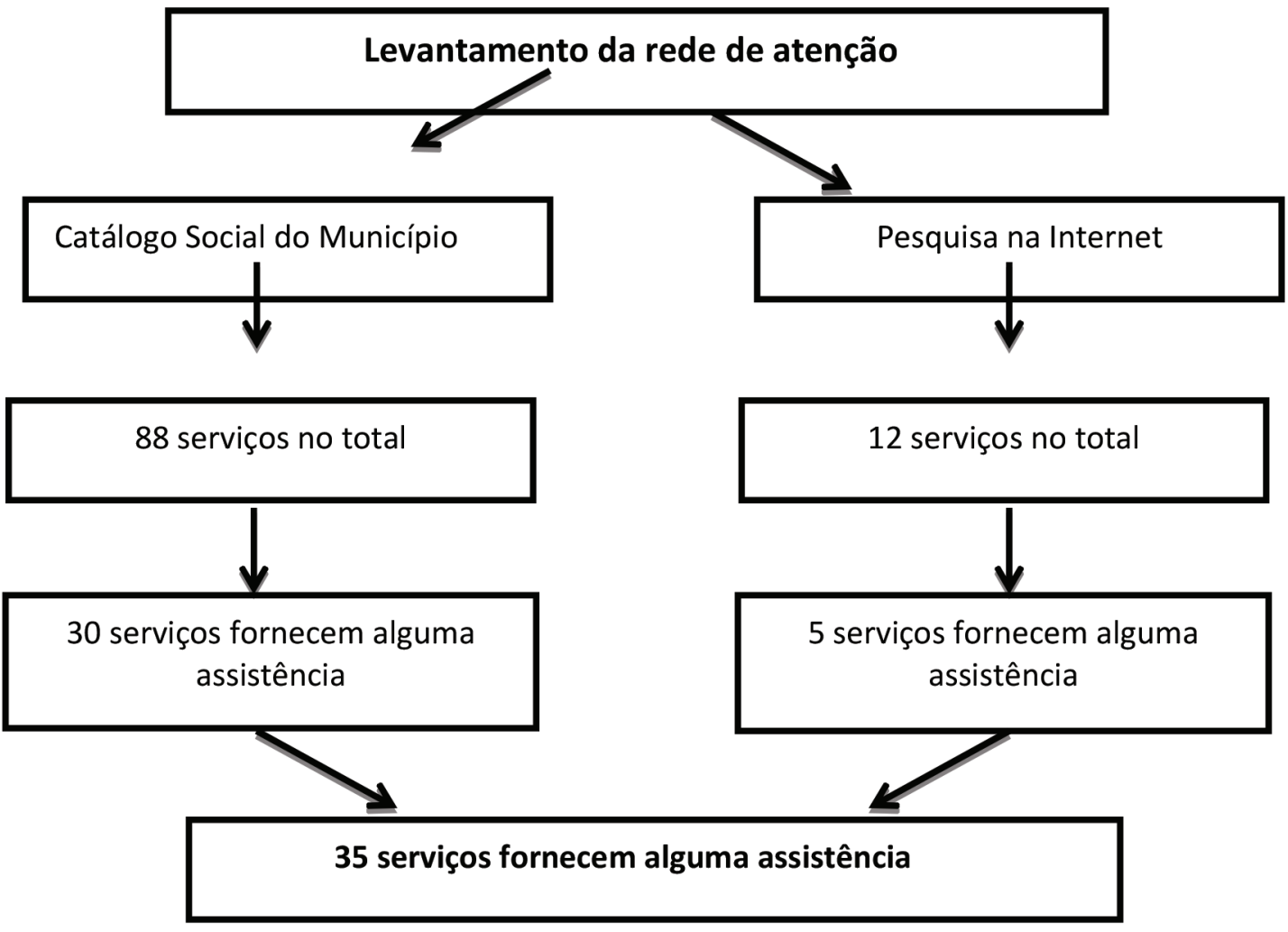

FONTE: elaborado pelos autores

Desses serviços encontrados, 45,7\% correspondem a serviços de saúde governamentais que atendem idosos e/ou cuidadores, mas não são específicos para essa população (Quadro 1). Foi observado um número expressivo de entidades religiosas, sendo aproximadamente $28 \%$ do total, que englobam a rede de atenção ao idoso.

Quadro 1 - Tipologia dos serviços que prestam assistência à população idosa da região Norte de Juiz de Fora, Minas Gerais

\begin{tabular}{|c|c|c|c|}
\hline Serviço & Tipo de serviço & $\mathbf{N}$ & $\%$ \\
\hline UPA & Governamental & 1 & $2,85 \%$ \\
\hline UAPS & Governamental & 14 & $40 \%$ \\
\hline CEO & Governamental & 1 & $2,85 \%$ \\
\hline CRAS & Governamental & 1 & $2,85 \%$ \\
\hline CREAS & Governamental & 1 & $2,85 \%$ \\
\hline Curumim & Governamental & 1 & $2,85 \%$ \\
\hline Escola & Governamental & 4 & $11,4 \%$ \\
\hline Associação de Moradores & Não Governamental & 1 & $2,85 \%$ \\
\hline Igreja Católica & Não Governamental & 6 & $17,25 \%$ \\
\hline
\end{tabular}


(Conclusão)

\begin{tabular}{|c|c|c|c|}
\hline Serviço & Tipo de serviço & $\mathbf{N}$ & $\mathbf{\%}$ \\
\hline Igreja Evangélica & Não Governamental & 2 & $5,7 \%$ \\
\hline Centro Espírita & Não Governamental & 2 & $5,7 \%$ \\
\hline CEU & Governamental & 1 & $2,85 \%$ \\
\hline \multicolumn{2}{r|}{ Total } & $\mathbf{3 5}$ & $\mathbf{1 0 0 \%}$ \\
\hline
\end{tabular}

FONTE: elaborado pelos autores

Dos serviços de saúde governamentais, as atividades ofertadas pelas 14 UAPS são destinadas para toda a população de sua área de abrangência e possuem serviços através de consultas agendadas, atendimento médico, visita domiciliar pela equipe e disponibilização de medicamentos oferecidos pelo SUS. Uma minoria realiza atividades em grupo, como para hipertensos, diabéticos e caminhada orientada, de acordo com a estruturação e gestão do serviço. Apenas uma UAPS não faz parte do PSF. Na região pesquisada, há apenas uma Unidade de Pronto Atendimento (UPA), ofertando atendimento de urgência e emergência para a população em geral, e um Centro de Especialidades Odontológicas (CEO), que disponibiliza serviços de mais complexidade para a população da região Norte da cidade.

Em relação aos serviços de assistência social governamentais, a atividade do CRAS, especificamente para o idoso, faz o cadastro para o Pró-Idoso - Programa de Atendimento à Terceira Idade da Prefeitura Municipal de Juiz de Fora e concede aos idosos o cadastro para ter a carteirinha de acesso ao transporte público gratuito. Além disso, o Centro de Referência Especializado de Assistência Social (CREAS) realiza ações preventivas, denúncias contra violência, atendimento às famílias em situação de vulnerabilidade e promoção dos direitos de toda a população da região. Apesar de ser voltado para ações infantis, encontrou-se um Curumim, onde é realizado um grupo de ginástica para pessoas com hipertensão arterial, não sendo focado somente para o público idoso. Algumas escolas estaduais oferecem à comunidade local atividades como: artesanato, ginástica, aula de violão, aula de dança e horta comunitária para a população geral, através de professores da instituição ou voluntários da região. Na região Norte está localizada uma praça onde está inserido o Centro de Artes e Esportes Unificados (CEU), que fornece artesanato, aula de teatro, aula de violão, caminhada orientada e ginástica para a comunidade. Oferece, ainda, aula de hip-hop e curso de informática que são específicos para idosos.

Dos serviços não governamentais, há apenas uma Associação de Moradores que disponibiliza aula de artesanato, podendo participar qualquer pessoa que reside no bairro onde se localiza a associação. Na região Norte foram achados seis serviços que são ofertados pelas Igrejas Católicas, com atividades que incluem os idosos, como 
ginástica para a comunidade, grupo de bordado, ponto cruz e crochê, atendimento fonoaudiólogo, atendimento psicológico, orientação sobre os benefícios e serviços do Instituto Nacional do Seguro Social (INSS), terapia comunitária, roda de terapia, pastoral da escuta e comunhão domiciliar. Uma igreja disponibiliza o espaço para o funcionamento do Pró-Idoso, onde são realizados grupos de dança, com atividade física e de ginástica. Além disso, foi encontrado um curso específico para cuidadores de idosos, oferecidos por um fisioterapeuta e um psicólogo. Dentre as Igrejas Evangélicas da região, duas ofertam orientações de advocacia para qualquer pessoa e evangelismo em ILPI para institucionalizados. Dois Centros Espíritas oferecem doações de alimentos e orientações espirituais para famílias carentes. Todos os serviços encontrados nesta pesquisa oferecem seus serviços de forma universal aos interessados, sendo assim, não são específicos para idosos.

A partir da localização dos serviços, foi criada uma conta de e-mail no Google (Gmail) e posteriormente o formulário on-line do Google Drive, contendo dados do questionário. Após o preenchimento do formulário, foi criada automaticamente uma planilha on-line no Google Fusion Tables, sendo possível a partir desta plotar os endereços dos serviços encontrados juntamente com as atividades prestadas em um mapa de localização (Figura 2) e em um mapa de calor (heatmap) (Figura 3) que demonstra uma dispersão dos serviços em todo o território norte da cidade.

Figura 2 - Mapa de localização dos serviços que oferecem assistência à população idosa da região Norte de Juiz de Fora, Minas Gerais

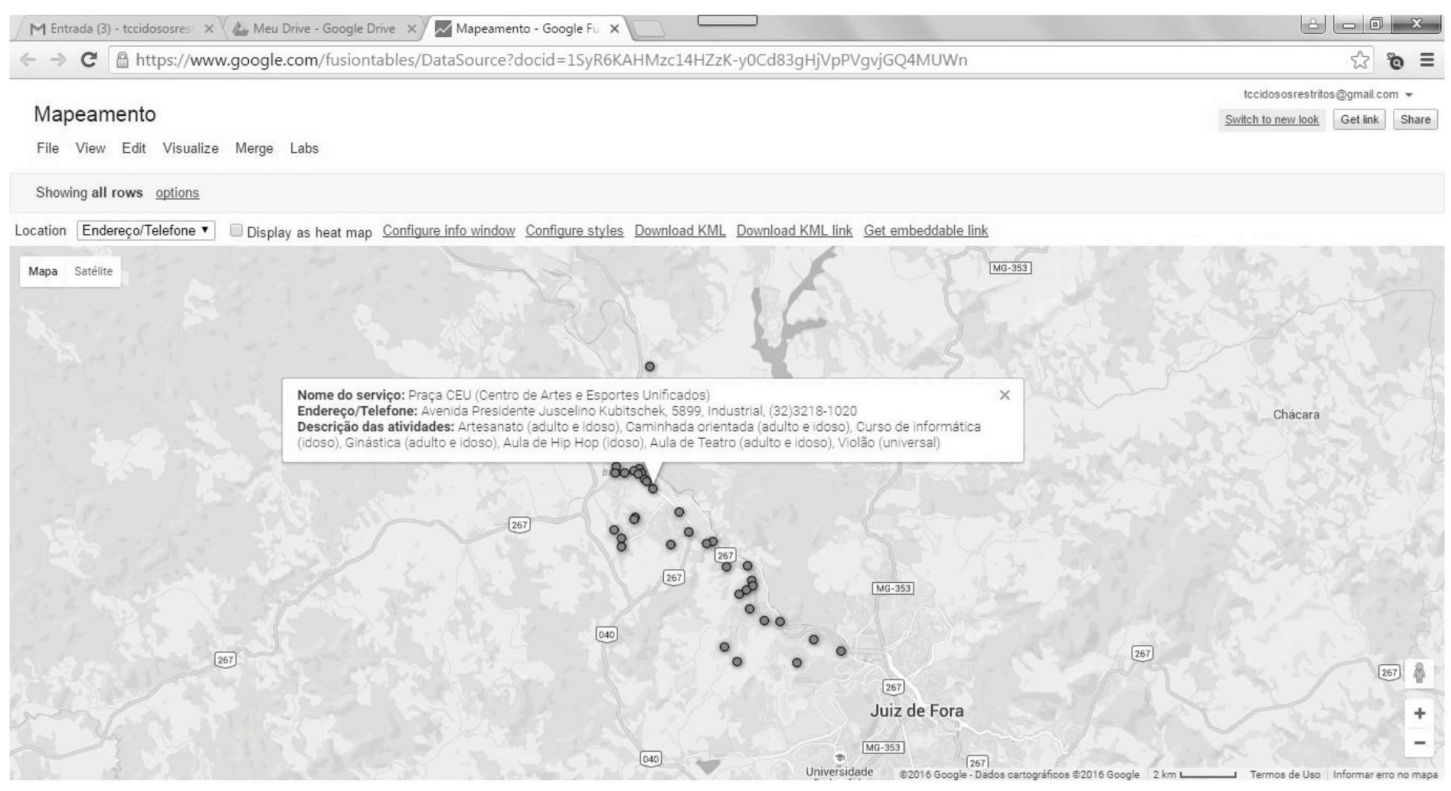

Fonte: disponível em <https://www.google.com/fusiontables/DataSource?snapid=S1822539_ zyh> 
Figura 3 - Mapa de calor (heatmap) dos serviços que oferecem assistência à população idosa da região Norte de Juiz de Fora, Minas Gerais

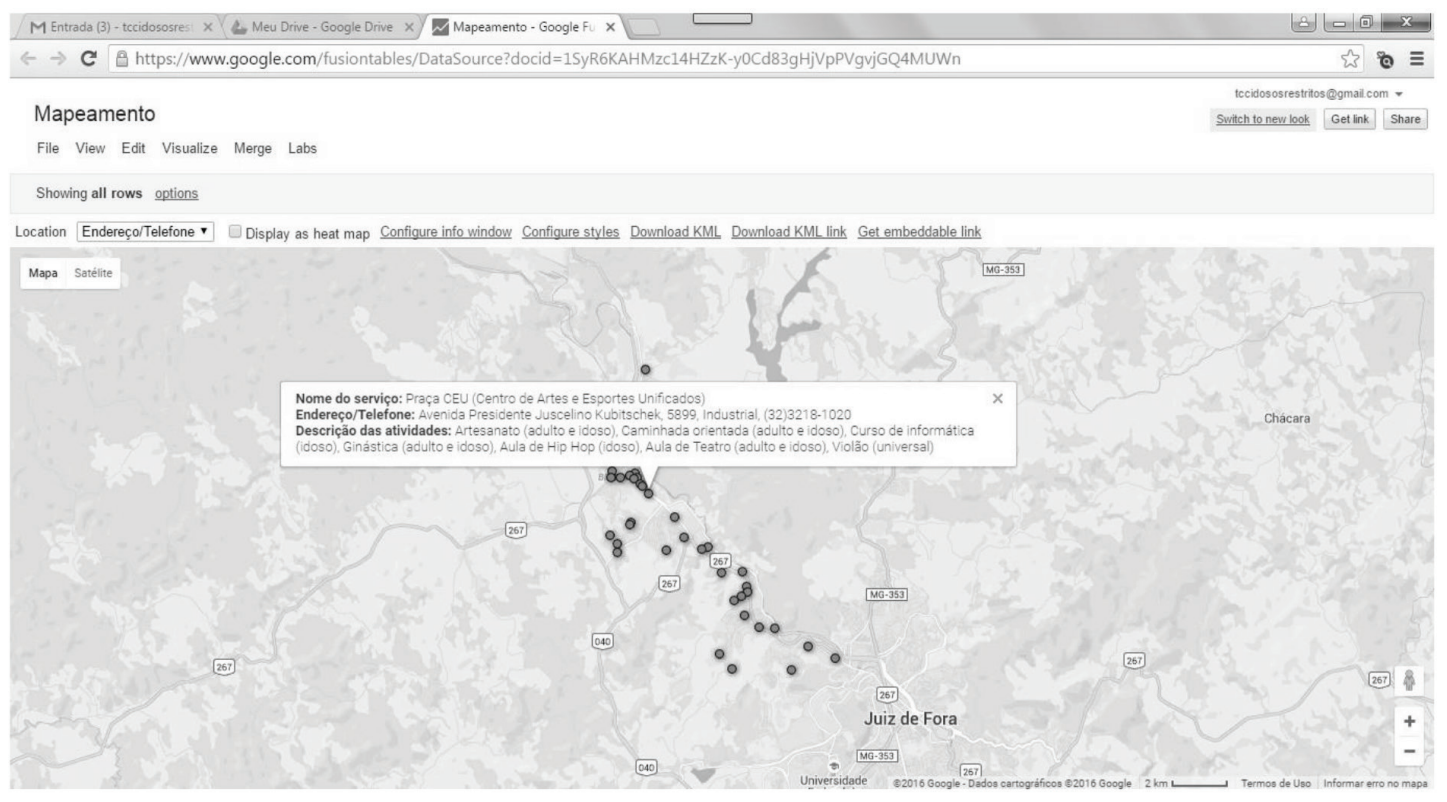

FONTE: Dos autores

\section{DISCUSSÃO}

Devido à alteração do contexto familiar e social em que o idoso está inserido, há uma demanda em aumentar o espectro de apoio, exigindo a participação de diversos setores e organizações da sociedade, para atender as necessidades tanto dos idosos quanto de seus cuidadores ${ }^{25,26}$. Nota-se a necessidade de uma rede de atenção específica para os idosos, que pode conter assistência protecionista de eventos prejudiciais, ações de saúde em forma de informações e recursos, e acesso a seus direitos ${ }^{27}$.

A partir da identificação dos 35 serviços encontrados, pode-se notar que a maioria era composta por serviços de saúde, coordenada pela atenção primária e com apenas um serviço secundário e um terciário, indicando a necessidade de um sistema que tenha a atenção primária como ordenadora e coordenadora dos outros serviços de saúde, como sugerido por Mendes ${ }^{20}$.

Há um predomínio de suporte de saúde classificado como formal, assim como os achados do estudo de Alvarenga et $a{ }^{21}{ }^{21}$ Foi observado que as atividades estão frequentemente voltadas para ações individuais, com pouco enfoque para a coletividade e para as ações de prevenção de agravos e promoção de bem-estar físico e emocional. No entanto, a maior parte das doenças que acometem os idosos precisa de cuidados mais especializados que fogem da capacidade da família, necessitando que a assistência prestada pelo sistema de saúde ocorra em situações crônicas, nas quais o cuidado adequado seria contínuo e somente potencializado por uma equipe multidisciplinar. 
Segundo Mendes ${ }^{20}$, isso se classifica como uma situação de incoerência entre os serviços de saúde ofertados e as transições demográficas e epidemiológicas pelo qual o Brasil vem passando.

Considerando que a maioria dos serviços de saúde encontrados são constituídos de UAPS, que ofertam atenção apenas em uma área de abrangência, pode-se concluir que essa instituição representa o único meio de acesso universal, considerando a grandeza do território da região Norte. Sendo assim, a visita domiciliar, frequente nas ações do PSF, deve ser vista como uma única possibilidade de assistência para algumas pessoas que possuem menor grau de funcionalidade ou restrição. A visita domiciliar deve ser valorizada, pois podem-se observar as características do indivíduo e de sua moradia, adequando a assistência ou orientações que podem envolver o paciente e/ou a família ${ }^{28}$.

Foi encontrado na região Norte da cidade um CRAS e um CREAS, sendo que há mais dois CRAS em fase de implantação, encontrados no Catálogo Social. O CRAS oferece proteção às famílias em situação de vulnerabilidade social e o CREAS dá um suporte especializado em situações individualizadas, nas quais o vínculo familiar já está rompido, sendo de extrema importância para qualquer região, principalmente as de baixo nível socioeconômico, além de a articulação entre os serviços ser um ponto positivo para a sociedade ${ }^{21}$.

O espaço das escolas públicas de ensino fundamental e médio oferece atividades de lazer e recreativa aos idosos, por meio de parcerias com empresas governamentais e não governamentais. Isso demonstra a importância da utilização de outros espaços públicos em prol da coletividade. Também neste sentido, as instituições religiosas contribuem para a assistência voluntária da região Norte da cidade, caracterizando uma prática comum entre a comunidade. Essa assistência é tradicionalmente prestada desde os primeiros séculos por seus membros ou fiéis, para aliviar os sofrimentos da população carente, constituindo uma ação caridosa que faz parte do dia a dia das pessoas, contribuindo para a rede de atenção social ${ }^{29}$. Os integrantes dessas ações, que podem ser individuais ou de instituições, são considerados sujeitos sociais, proporcionando alterações nas situações do cotidiano e na realidade social, o que deveria ser de responsabilidade do Estado, que se livra da pressão exercida pela sociedade, apropriando-se da cooperação solidária ${ }^{30}$. Caberia ao Estado a manutenção dessa rede de atenção, colocando em prática os direitos da população, sendo os serviços voluntários apenas um suporte. Conforme Silva ${ }^{30}$, o Estado diminui a oferta de orçamentos e funcionários destinados às políticas sociais, repassando essa incumbência para a sociedade civil.

Nesse contexto, os serviços da rede de apoio podem ser considerados fundamentais para a proteção e manutenção da saúde e do bem-estar dos indivíduos ${ }^{27}$. Vale ressaltar que as características da população idosa e da região influenciam na busca por informações e utilização dos serviços. A partir da realidade encontrada no Brasil, grande parte da população possui dificuldades de sobrevivência, baixo nível 
socioeconômico e educacional, gerando uma menor mobilização da população e da busca pelos interesses comuns da sociedade civil ${ }^{31}$.

\section{CONSIDERAÇÕES FINAIS}

A partir do mapeamento realizado, observa-se que os serviços da região Norte de Juiz de Fora estão caminhando para uma estruturação da rede de atenção à população idosa, englobando instituições governamentais e não governamentais, que oferecem suporte mais focado ao idoso com doenças agudas e/ou crônicas, mas que ainda possuem um bom nível de funcionalidade; porém, os idosos com algum tipo de restrição não possuem o devido enfoque pela rede de assistência localizada. Os idosos restritos, principalmente os que vivem sozinhos, estando eles com restrição funcional, deveriam ser o centro de atenção da equipe de saúde e, com certeza, precisariam de serviços formais e informais para o cuidado.

Nesse contexto, a atenção primária deve estar estruturada e preparada para atender a demanda emergente da população de idosos, através de uma equipe multiprofissional e capacitada, que atenda a comunidade que possui doenças já instaladas ou que previnam os acometimentos, diminuindo assim a demanda de atenção às morbidades, gerando maior qualidade de vida. Como a principal forma de promoção da saúde, tem-se a educação em saúde, que pode fornecer informações para o idoso, seus familiares e os cuidadores, que merecem atenção especial dos serviços, tanto do âmbito social quanto de saúde, pois são estes os responsáveis por tempo integral dos idosos. Deve-se enfatizar o auxílio sobre o autocuidado em idosos "mais jovens" e aqueles que não possuem cuidador ou vivem sozinhos.

A prevenção de agravos, considerada uma ação intersetorial, cujas ações extrapolam o setor saúde, mas agregam ainda os setores de transporte, obras/ infraestrutura, educação, seguridade social/previdência e outros, deve ser planejada de forma integrada, para que a resposta à qualidade de vida de uma população seja percebida com impacto epidemiológico.

Espera-se que este estudo possa contribuir para futuros trabalhos que mobilizem a sociedade organizada e o Estado, no sentido de dar respostas às necessidades da população idosa, que tende a crescer ainda mais. Que as equipes que prestam cuidado a população, tanto governamentais como não governamentais, tenham clareza de que existe uma população com dificuldade de locomoção que está negligenciada pelos serviços. 


\section{REFERÊNCIAS}

1. Nunes DP, Nakatani AY, Silveira EA, Bachion MM, Souza MR. Capacidade funcional, condições socioeconômicas e de saúde de idosos atendidos por equipes de Saúde da Família de Goiânia (GO, Brasil). Cien Saude Colet. 2010; 15(6):2887-98.

2. Ministério da Saúde (BR). Departamento de Atenção Básica. Envelhecimento e saúde da pessoa idosa. Brasília: Ministério da Saúde; 2006.

3. Santana RLF, Pouchain GC, Bissi LF. A Previdência Social e o Censo 2000: Perfil dos Idosos. Ministério da Previdência e Assistência Social. Secretaria de Previdência Social. 2002 set; 14(9).

4. Agustini FC. Introdução ao direito do idoso. Florianópolis: Fundação Boiteux; 2003.

5. Victor JF, Ximenes LB, Almeida PC, Vasconcelos FF. Perfil sociodemográfico e clínico de idosos atendidos em Unidade Básica de Saúde da Família. Acta Paul Enferm. 2009 ago; 22(1):49-54.

6. IBGE. Síntese de Indicadores Sociais: Uma análise das condições de vida da população brasileira 2014. Rio de Janeiro: Ministério do Planejamento, Orçamento e Gestão; 2014.

7. UFJF. Relatório Executivo "Diagnóstico socioeconômico da população idosa de Juiz de Fora". Juiz de Fora: Pró-reitoria de Extensão; 2012.

8. Ministério da Saúde (BR). Envelhecimento e saúde da pessoa idosa. Série A. Normas e Manuais Técnicos. Brasília: Ministério da Saúde; 2007.

9. Fonseca HLA. Caracterização dos principais problemas de saúde do idoso acamado, e desafios para a ESF no acompanhamento destes [monografia]. Belo Horizonte: Universidade Federal de Minas Gerais; 2010.

10. Uesugui HM, Fagundes DS, Pinho DLM. Perfil e grau de dependência de idosos e sobrecarga de seus cuidadores. Acta Paul Enferm. 2011 mar; 24(5):689-94.

11. Ursine PGS, Cordeiro HA, Moraes CL. Prevalência de idosos restritos ao domicílio em região metropolitana de Belo Horizonte (Minas Gerais, Brasil). Cien Saude Colet. 2011; 16(6):2953-62.

12. Caldas CP. Envelhecimento com dependência: responsabilidades e demandas da família. Cad Saude Publica. 2003 maio; 19(3):773-81.

13. Figueiredo M LF, Luz MHBA, Brito CMS, Sousa SNS, Silva DRS. Diagnósticos de enfermagem do idoso acamado no domicílio. Rev Bras Enferm. 2008 jun; 61(4):464-9.

14. Maria Nunes L, Portella MR. O idoso fragilizado no domicílio: a problemática encontrada na Atenção Básica em Saúde. Boletim de Saúde, Porto Alegre. 2003 jul; 17(2). 
15. Nakatani AYK, Silva LB, Bachion MM, Nunes DP. Capacidade funcional em idosos na comunidade e propostas de intervenções pela equipe de saúde. Rev Eletrônica Enferm. 2009 mar; 11(1):144-50.

16. Bezerra MIC, Lima MJMR, Lima YCP. A visita domiciliar como ferramenta de cuidado da fisioterapia na Estratégia Saúde da Família. SANARE, Sobral. 2015 jan; 14(1):7680 .

17. Ricci NA, Kubota MT, Cordeiro RC. Concordância de observações sobre a capacidade funcional de idosos em assistência domiciliar. Rev Saude Publica. 2005 fev; 39(4):655-62.

18. Chaimowicz FA. Saúde dos idosos brasileiros às vésperas do século XXI: problemas, projeções e alternativas. Rev Saude Publica. 1997 abr; 31(2):184-200.

19. Cesar JA, Oliveira-Filho JA, Bess G, Cegielka R, Machado J, Gonçalves TS, et al. Perfil dos idosos residentes em dois municípios pobres das regiões Norte e Nordeste do Brasil: resultados de estudo transversal de base populacional. Cad Saude Publica. 2008 ago; 24(8):1835-45.

20. Mendes EV. As redes de atenção à saúde. Ciência e Saúde Coletiva. 2010; 15(5):2297-305.

21. Alvarenga MRM, Oliveira MAC, Domingues MAR, Amendola F, Faccenda O. Rede de suporte social do idoso atendido por equipes de Saúde da Família. Cien Saude Colet. 2011; 16(5):2603-11.

22. Juiz de Fora. Mapas de Juiz de Fora - Região Norte [mapa]. Prefeitura de Juiz de Fora. [2016 maio 20]. Disponível em: https://www.pjf.mg.gov.br/cidade/mapas/ mapa_norte.php.

23. Prefeitura (Juiz de Fora). Catálogo social - Juiz de Fora 2012. Juiz de Fora: Secretaria de Assistência Social - Funalfa; 2012.

24. Costa PHA. Mapeamento da Rede de Atenção aos Usuários de Droga: um estudo exploratório [dissertação]. Juiz de Fora: Universidade Federal de Juiz de Fora; 2014.

25. Mota FRN, Oliveira ET, Marques MB, Bessa MEP, Leite BMB, Silva MJ. Família e redes sociais de apoio para o atendimento das demandas de saúde do idoso. Esc Anna Nery. 2010 out; 14(4):833-8.

26. Nardi EFR, Oliveira MLF. Conhecendo o apoio social ao cuidador familiar do idoso dependente. Rev Gaucha Enferm. 2008 mar; 29(1):47-53.

27. Brito TRP, Costa RS, Pavarini SCL. Idosos com alteração cognitiva em contexto de pobreza: studando a rede de apoio social. Rev Esc Enferm USP. 2012; 46(4):906-13.

28. Viana SO, Alvarenga JP, Camargos MMM, Taciano MAR, Rafael MA. Perfil dos indivíduos avaliados em domicílio pela fisioterapia nas unidades básicas de saúde de Betim. Rev APS. 2013 jul; 16(3):278-86. 
29. Kuhn, W. A Prática da Caridade e da Beneficência Social na Igreja Cristã. Foco na Pessoa. 2013 jun; 2(2):40-50.

30. Silva CN. Igreja católica, assistência social e caridade: aproximações e divergências. Sociologias. 2006 jan; 8(15):326-51.

31. Andrade GRB, Vaitsman J. Apoio social e redes: conectando solidariedade e saúde. Ciência e Saúde Coletiva. 2002; 7(4):925-34.

Submissão: outubro de 2017. Aprovação: maio de 2019. 